\title{
Axion dark matter search using the storage ring EDM method
}

\author{
Seung Pyo Chang, ${ }^{a, b}$ Selcuk Haciomeroglu, ${ }^{a}$ On Kim, ${ }^{a, b}$ Soohyung Lee, ${ }^{a}$ Seongtae \\ Park ${ }^{* a}$ Yannis K. Semertzidis ${ }^{a, b}$ \\ ${ }^{a}$ Center for Axion and Precision Physics Research, IBS, Daejeon 34051, Republic of Korea \\ ${ }^{b}$ Department of Physics, KAIST, Daejeon 34141, Republic of Korea \\ E-mail: stpark@ibs.re.kr
}

\begin{abstract}
We propose using a modified storage ring EDM method to search for the axion dark matter induced EDM oscillation in nucleons. The method uses a combination of B and E-fields to produce a resonance between the $g-2$ precession frequency and the background axion field oscillation to greatly enhance the sensitivity to it. An axion frequency range of $10^{-9} \mathrm{~Hz}$ to $100 \mathrm{MHz}$ can be scanned with high sensitivity, corresponding to $f_{a}$ range of $10^{13} \mathrm{GeV} \leq f_{a} \leq 10^{30} \mathrm{GeV}$ the breakdown scale of the global symmetry generating the axion or axion like particles (ALPs).
\end{abstract}

XVII International Workshop on Polarized Sources, Targets \& Polarimetry

16-20 October 2017

Kaist, South Korea

${ }^{*}$ Speaker. 


\section{Introduction}

An axion in the parameter range of $10^{11} \mathrm{GeV} \leq f_{a} \leq 10^{13} \mathrm{GeV}$ is potentially observable using microwave cavity resonators, where $f_{a}$ is the axion decay constant $[1,2,3]$. This method detects photons from the axion dark matter conversion in the presence of strong magnetic fields. In the next decade it is expected that the axion frequency range of $0.1-50 \mathrm{GHz}$ may be covered using microwave and/or open cavity resonators. However, this method cannot be used for higher value region of the scale $f_{a}$ (lower mass region) because the axion-photon coupling is suppressed by $f_{a}\left(\sim 1 / f_{a}^{2}\right)$ and the required resonance structures would be impractically large. For the higher values of the scale, including $M_{\mathrm{GUT}}\left(\sim 10^{16} \mathrm{GeV}\right)-M_{\mathrm{PL}}\left(\sim 10^{19} \mathrm{GeV}\right)$, axion-gluon coupling can be considered, which gives a time varying electric dipole moment (EDM) to nucleons. For example, in the nucleon case, the EDM can be expressed as $[2,3]$

$$
\begin{gathered}
d_{n}=2.4 \times 10^{-16} \frac{a}{f_{a}} \sim\left(9 \times 10^{-35}\right) \cos \left(m_{a} t\right) \quad[e \cdot \mathrm{cm}], \\
a(t)=a_{0} \cos \left(m_{a} t\right),
\end{gathered}
$$

where $a(t)$ is the axion dark matter field and $m_{a}$ is the axion mass. By combining the eq.(1.1) and eq.(1.2) with a possible static EDM, one can write the total EDM as,

$$
d(t)=d_{D C}+d_{A C} \cos \left(m_{a} t+\varphi_{x}\right),
$$

where $d_{D C}$ and $d_{A C}$ are the magnitudes of the static and oscillating part of EDM, respectively, and $\varphi_{x}$ is the initial phase of the axion field. In this paper, we propose using the storage ring technique to probe the oscillating EDM signal [5,6]. This method is expected to be more sensitive and the systematic errors are easier to handle than in the frozen spin storage ring EDM method. Using the storage ring method, one can scan the frequency range from $10^{-9} \mathrm{~Hz}$ up to $100 \mathrm{MHz}$, which corresponds to an axion parameter space of about $10^{13} \mathrm{GeV} \leq f_{a} \leq 10^{30} \mathrm{GeV}$.

\section{Resonance of Axion induced oscillating EDM with $g-2$ precession in the storage ring}

The previously proposed storage ring EDM experiment is optimized for a DC (fixed in time) nucleon EDM, applied to protons and deuterons. It is designed to keep (freeze) the particle spin direction in the longitudinal direction for the duration of the storage time, typically for $10^{3} \mathrm{~s}$, the stored beam polarization coherence time. In this case, the radial electric field in the particle rest frame is precessing the particle spin in the vertical plane. The precession frequency in the presence of both $\mathrm{E}$ and $\mathrm{B}$ fields is expressed by the T-BMT equation eq. (2.1) $\sim(2.3)[7,8]$.

$$
\begin{gathered}
\vec{\omega}=\vec{\omega}_{a}+\vec{\omega}_{d}, \\
\vec{\omega}_{a}=-\frac{e}{m}\left[a \vec{B}-\left(a-\frac{1}{\gamma^{2}-1}\right) \frac{\vec{\beta} \times \vec{E}}{c}\right],
\end{gathered}
$$




$$
\vec{\omega}_{d}=-\frac{e}{m}\left[\frac{\eta}{2}\left(\frac{\vec{E}}{c}+\vec{\beta} \times \vec{B}\right)\right],
$$

where $a=(g-2) / 2$ is the magnetic anomaly with $a=-0.14$ for deuteron. The parameter $\eta$ shown in the equation is related to the electric dipole moment $d$ as $d=\eta e \hbar / 4 m c$. Since we are dealing with a time varying EDM due to the oscillating axion background field, $\eta$ is also a function of time. The $\vec{\omega}_{a}$ is the angular frequency, i.e., $2 \pi$ times the $g-2$ frequency, describing the spin precession in the horizontal plane relative to the momentum precession.

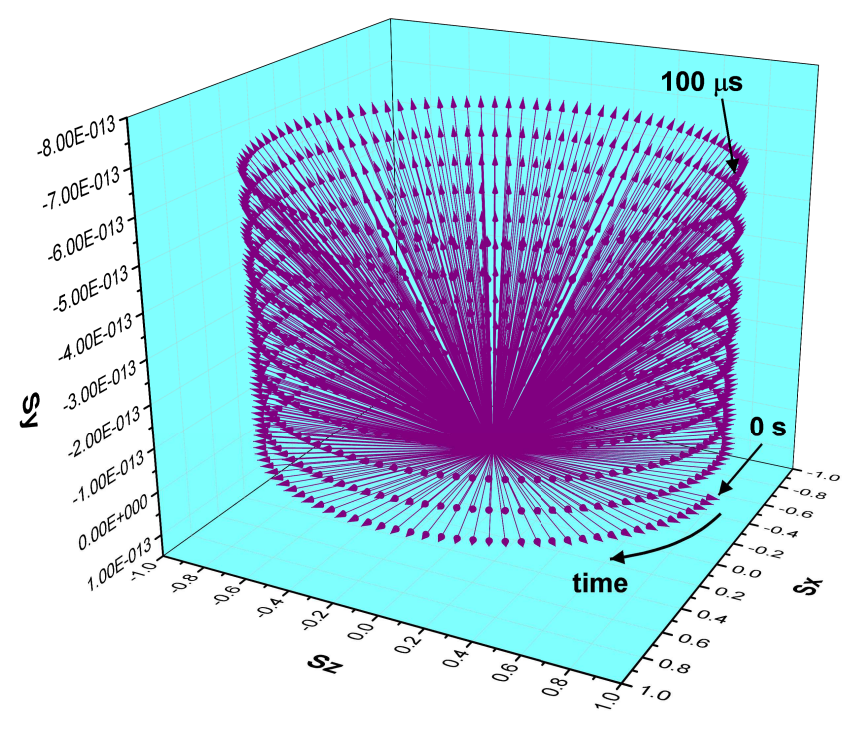

Figure 1: Deuteron spin precession in E/B combined ring.

The term $\vec{\omega}_{d}$ is due to the EDM and the corresponding precession takes place in the vertical plane. For a time independent nucleon EDM, the spin vector will precess vertically for the duration of the storage time if the horizontal spin component is fixed to the momentum direction. This condition can be achieved by setting the E and B fields properly and is called the frozen spin condition.

With a nonzero $g-2$ frequency, the average EDM precession angle becomes zero for the static EDM case because the relative $\mathrm{E}$ field direction to the spin vector changes within every cycle of $g-2$ precession. For example, the spin tilts in one direction (up or down) due to the EDM within one half cycle and then tilts in the opposite direction for the other half cycle, resulting in an average accumulation of zero. The presence of a static EDM will only slightly tilt the $g-2$ precession plane away from the horizontal plane, without a vertical spin accumulation. In contrast, for an oscillating $\mathrm{EDM}$, when the axion frequency $\left(\omega_{x}\right)$ is the same as the $g-2$ frequency with the appropriate phase, the precession angle can be accumulated in one direction. This is possible because the EDM flips every half cycle and the relative direction between the E field and the EDM $d$ always remains the same. 
In this idea of resonant axion induced EDM with $g-2$ spin precession, one can utilize the strong effective electric field $\vec{E}^{*}=\vec{E}+c \vec{\beta} \times \vec{B}$, which comes from the B field due to particle motion. In this case, the effective electric field is about one or two orders of magnitude larger than the applied external E field which can be up to $10 \mathrm{MV} / \mathrm{m}$ and has an apparent technical limitation in strength.

Fig. 1 shows a simulation result for deuteron spin precession in a $\mathrm{E} / \mathrm{B}$ combined ring with $100 \mathrm{kHz}$ of g-2 frequency. The electric field and magnetic field used in the simulation were $7.05 \times$ $10^{6} \mathrm{~V} / \mathrm{m}$ and $0.38 \mathrm{~T}$, respectively. The initial spin direction was set to the $+\mathrm{z}$ direction $(0,0,1)$ and the total precession time shown in the figure is $100 \mu \mathrm{s}$. As can be seen, the vertical spin component $\left(S_{y}\right)$ is accumulated while the horizontal spin precession takes place at the g-2 frequency. As mentioned before, the vertical precession rate depends on the initial axion phase.

\section{Sensitivity of the experiment}

In this study, we use the following method to calculate the sensitivities for the axion EDM measurement including the oscillation effect. First we chose the target axion frequency and then calculate the corresponding E and B-fields for the particle storage which give the same $g-2$ frequency as the chosen axion frequency. Then, the axion oscillation and $g-2$ precession will be on resonance and the EDM precession angle in the vertical plane can keep accumulating during the measurement time. With the chosen axion frequency and the axion quality factor $Q$, we estimated the statistical error and the resulting error was used to calculate the experiment sensitivity along with the effective electric field. This method can be used not only for the nucleons like deuteron or protons but also can be used for other leptonic particles like muons provided there is a coupling between the oscillating $\theta_{Q C D}$ induced by the background axion dark matter field and the particle EDM. As shown in eq. (2.3), the EDM part of the precession rate can be rewritten as eq. (3.1)

$$
\omega_{d}=\frac{d \theta}{d t}=-\frac{2 d}{\hbar} E^{*}, E^{*}=E+c \beta B .
$$

Accordingly, $d=\frac{\hbar}{2 E^{*}} \omega_{d}$, and the error for the $\operatorname{EDM} d$ can be written as eq. (3.2).

$$
\sigma_{d}=\frac{\hbar}{2 E^{*}} \sigma_{\omega_{d}},
$$

where $\sigma_{\omega_{d}}$ is the error for $\omega_{d}$. It can be obtained from the fit of the vertical precession angle $\theta$ as a function of time. For this study, we wrote a simulation program that produces asymmetry data $\varepsilon(t)$ which gives precession angle $\theta$ as a function of time. The left-right asymmetry $\varepsilon$ for the vertically polarized beam is defined as follows [9].

$$
\varepsilon(t)=\frac{L-R}{L+R}(t)=P A \theta(t),
$$

where $A$ is the analyzing power, $\theta(t)$ is the accumulated EDM precession angle in the vertical plane and $L$ and $R$ are number of hits on the left and right detectors, respectively. We use this formula in the MC simulation to get the asymmetry data with given axion frequency.

Table 1 and 2 show sensitivity calculation results for both proton and deuteron cases. 
Table 1: Examples of experimental parameters for frequency tuning, and results of sensitivity calculation (Deuteron). The analyzing power was assumed to be $A=0.36$ if the momentum $\mathrm{P}$ was below $2 \mathrm{GeV} / c$ and $\mathrm{A}=0.15$ was used for the momentum $\mathrm{P}>2 \mathrm{GeV} / c$. The ring bending radius was $10 \mathrm{~m}$. The polarimeter efficiency was assumed to be $2 \%$ and initial polarization was 0.8 . The axion quality factors: $Q_{a x 1}=3 \times 10^{6}, Q_{a x 2}=10^{10}$.

\begin{tabular}{|c|c|c|c|c|c|c|c|c|c|c|}
\hline \multirow{2}{*}{$\mathrm{B}(\mathrm{T})$} & \multirow{2}{*}{$\mathrm{P}(\mathrm{GeV} / \mathrm{c})$} & \multirow{2}{*}{$f_{g-2}$} & \multirow{2}{*}{$\mathrm{E}_{\mathrm{r}}(\mathrm{V} / \mathrm{m})$} & \multirow{2}{*}{$\mathrm{E}^{*}(\mathrm{~V} / \mathrm{m})$} & \multicolumn{6}{|c|}{ Sensitivity $(\mathrm{e} \cdot \mathrm{cm})$} \\
\cline { 6 - 11 } & & & & & \multicolumn{2}{|c|}{$S C T=10^{3} s$} & \multicolumn{2}{|c|}{$S C T=10^{4} s$} & \multicolumn{2}{c|}{$S C T=10^{5} s$} \\
\cline { 6 - 11 } & & & & $Q_{a x 1}$ & $Q_{a x 2}$ & $Q_{a x 1}$ & $Q_{a x 2}$ & $Q_{a x 1}$ & $Q_{a x 2}$ \\
\hline 0.3800 & 0.9428 & $1 . \mathrm{E} 1$ & $8.82 \mathrm{E} 6$ & $4.23 \mathrm{E} 7$ & $9.9 \mathrm{E}-31$ & $9.9 \mathrm{E}-31$ & $3.1 \mathrm{E}-31$ & $3.1 \mathrm{E}-31$ & $9.9 \mathrm{E}-32$ & $9.9 \mathrm{E}-32$ \\
\hline 0.3800 & 0.9429 & $1 . \mathrm{E} 2$ & $8.82 \mathrm{E} 6$ & $4.23 \mathrm{E} 7$ & $9.9 \mathrm{E}-31$ & $9.9 \mathrm{E}-31$ & $3.1 \mathrm{E}-31$ & $3.1 \mathrm{E}-31$ & $1.4 \mathrm{E}-31$ & $9.9 \mathrm{E}-32$ \\
\hline 0.3800 & 0.9433 & $1 . \mathrm{E} 3$ & $8.80 \mathrm{E} 6$ & $4.24 \mathrm{E} 7$ & $9.9 \mathrm{E}-31$ & $9.9 \mathrm{E}-31$ & $4.3 \mathrm{E}-31$ & $3.1 \mathrm{E}-31$ & $3.8 \mathrm{E}-31$ & $9.9 \mathrm{E}-32$ \\
\hline 0.3800 & 0.9473 & $1 . \mathrm{E} 4$ & $8.65 \mathrm{E} 6$ & $4.27 \mathrm{E} 7$ & $1.4 \mathrm{E}-30$ & $9.9 \mathrm{E}-31$ & $8.3 \mathrm{E}-31$ & $3.1 \mathrm{E}-31$ & $1.2 \mathrm{E}-30$ & $9.9 \mathrm{E}-32$ \\
\hline 0.3800 & 0.9880 & $1 . \mathrm{E} 5$ & $7.05 \mathrm{E} 6$ & $4.60 \mathrm{E} 7$ & $3.5 \mathrm{E}-30$ & $9.1 \mathrm{E}-31$ & $3.5 \mathrm{E}-30$ & $2.9 \mathrm{E}-31$ & $3.5 \mathrm{E}-30$ & $9.1 \mathrm{E}-32$ \\
\hline 0.3800 & 1.0345 & $2 . \mathrm{E} 5$ & $5.06 \mathrm{E} 6$ & $5.00 \mathrm{E} 7$ & $4.6 \mathrm{E}-30$ & $8.4 \mathrm{E}-31$ & $4.5 \mathrm{E}-30$ & $2.7 \mathrm{E}-31$ & $4.5 \mathrm{E}-30$ & $9.8 \mathrm{E}-32$ \\
\hline 0.3800 & 1.1326 & $4 . \mathrm{E} 5$ & $3.47 \mathrm{E} 5$ & $5.85 \mathrm{E} 7$ & $5.5 \mathrm{E}-30$ & $7.2 \mathrm{E}-31$ & $5.5 \mathrm{E}-30$ & $2.3 \mathrm{E}-31$ & $5.5 \mathrm{E}-30$ & $5.2 \mathrm{E}-32$ \\
\hline 0.3800 & 1.2386 & $6 . \mathrm{E} 5$ & $-5.47 \mathrm{E} 6$ & $6.82 \mathrm{E} 7$ & $5.8 \mathrm{E}-30$ & $6.2 \mathrm{E}-31$ & $5.3 \mathrm{E}-30$ & $2.0 \mathrm{E}-31$ & $5.8 \mathrm{E}-30$ & $1.1 \mathrm{E}-31$ \\
\hline 0.3800 & 1.3546 & $8 . \mathrm{E} 5$ & $-1.26 \mathrm{E} 7$ & $7.93 \mathrm{E} 7$ & $5.7 \mathrm{E}-30$ & $5.3 \mathrm{E}-31$ & $3.9 \mathrm{E}-30$ & $1.7 \mathrm{E}-31$ & $5.7 \mathrm{E}-30$ & $1.0 \mathrm{E}-31$ \\
\hline 0.3800 & 1.4836 & $1 . \mathrm{E} 6$ & $-2.14 \mathrm{E} 7$ & $9.20 \mathrm{E} 7$ & $5.5 \mathrm{E}-30$ & $4.6 \mathrm{E}-31$ & $3.5 \mathrm{E}-30$ & $1.4 \mathrm{E}-31$ & $5.5 \mathrm{E}-30$ & $1.0 \mathrm{E}-31$ \\
\hline 0.8000 & 2.5124 & $1 . \mathrm{E} 6$ & $-9.13 \mathrm{E} 6$ & $2.01 \mathrm{E} 8$ & $1.6 \mathrm{E}-30$ & $1.5 \mathrm{E}-31$ & $2.5 \mathrm{E}-30$ & $6.6 \mathrm{E}-32$ & $2.5 \mathrm{E}-30$ & $4.6 \mathrm{E}-32$ \\
\hline 0.9198 & 2.7574 & $1 . \mathrm{E} 6$ & 0.0 & $2.28 \mathrm{E} 8$ & $5.3 \mathrm{E}-30$ & $4.4 \mathrm{E}-31$ & $3.4 \mathrm{E}-30$ & $1.4 \mathrm{E}-31$ & $5.3 \mathrm{E}-30$ & $9.7 \mathrm{E}-32$ \\
\hline 9.1977 & 27.5740 & $1 . \mathrm{E} 7$ & 0.0 & $2.75 \mathrm{E} 9$ & $3.3 \mathrm{E}-30$ & $3.7 \mathrm{E}-32$ & $4.4 \mathrm{E}-30$ & $2.5 \mathrm{E}-32$ & $4.4 \mathrm{E}-30$ & $2.4 \mathrm{E}-32$ \\
\hline
\end{tabular}

Table 2: Examples of experimental parameters for frequency tuning, and results of sensitivity calculation (Proton). The analyzing power was assumed to be $A=0.6$ for the momentum $\mathrm{P}<1$ $\mathrm{GeV} / c$ and $A=0.25$ was used for the momentum $\mathrm{P}>1 \mathrm{GeV} / c$. The ring bending radius was $52 \mathrm{~m}$ for the E/B combined ring, and $r=10 \mathrm{~m}$ for the pure magnetic ring. The polarimeter efficiency used was $2 \%$ and initial polarization was 0.8 . The axion quality factors: $Q_{a x 1}=3 \times 10^{6}, Q_{a x 2}=10^{10}$.

\begin{tabular}{|c|c|c|c|c|c|c|c|c|c|c|}
\hline \multirow{2}{*}{$\mathrm{B}(\mathrm{T})$} & \multirow{2}{*}{$\mathrm{P}(\mathrm{GeV} / \mathrm{c})$} & \multirow{2}{*}{$f_{g-2}$} & \multirow{2}{*}{$\mathrm{E}_{\mathrm{r}}(\mathrm{V} / \mathrm{m})$} & \multirow{2}{*}{$\mathrm{E}^{*}(\mathrm{~V} / \mathrm{m})$} & \multicolumn{5}{|c|}{$S e n s i t i v i t y(\mathrm{e} \cdot \mathrm{cm})$} \\
\cline { 5 - 10 } & & & & & \multicolumn{2}{|c|}{$S C T=10^{3} s$} & \multicolumn{2}{|c|}{$S C T=10^{4} s$} & \multicolumn{2}{c|}{$S C T=10^{5} s$} \\
\hline & & & & $Q_{a x 1}$ & $Q_{a x 2}$ & $Q_{a x 1}$ & $Q_{a x 2}$ & $Q_{a x 1}$ & $Q_{a x 2}$ \\
\hline 0.00011 & 0.6984 & $1 . \mathrm{E} 1$ & $-8.0 \mathrm{E} 6$ & $8.02 \mathrm{E} 6$ & $1.6 \mathrm{E}-30$ & $1.6 \mathrm{E}-30$ & $5.0 \mathrm{E}-31$ & $5.0 \mathrm{E}-31$ & $1.6 \mathrm{E}-31$ & $1.6 \mathrm{E}-31$ \\
\hline 0.00010 & 0.6984 & $1 . \mathrm{E} 2$ & $-8.0 \mathrm{E} 6$ & $8.02 \mathrm{E} 6$ & $1.6 \mathrm{E}-30$ & $1.6 \mathrm{E}-30$ & $3.6 \mathrm{E}-31$ & $3.6 \mathrm{E}-31$ & $2.2 \mathrm{E}-31$ & $1.6 \mathrm{E}-31$ \\
\hline 0.00008 & 0.6982 & $1 . \mathrm{E} 3$ & $-8.0 \mathrm{E} 6$ & $8.01 \mathrm{E} 6$ & $1.6 \mathrm{E}-30$ & $1.6 \mathrm{E}-30$ & $6.9 \mathrm{E}-31$ & $5.0 \mathrm{E}-31$ & $6.1 \mathrm{E}-31$ & $1.6 \mathrm{E}-31$ \\
\hline-0.00015 & 0.6960 & $1 . \mathrm{E} 4$ & $-8.0 \mathrm{E} 6$ & $7.97 \mathrm{E} 6$ & $2.2 \mathrm{E}-30$ & $1.6 \mathrm{E}-30$ & $1.9 \mathrm{E}-30$ & $4.1 \mathrm{E}-31$ & $1.9 \mathrm{E}-30$ & $1.6 \mathrm{E}-31$ \\
\hline-0.00243 & 0.6747 & $1 . \mathrm{E} 5$ & $-8.0 \mathrm{E} 6$ & $7.57 \mathrm{E} 6$ & $6.4 \mathrm{E}-30$ & $1.7 \mathrm{E}-30$ & $4.9 \mathrm{E}-30$ & $5.3 \mathrm{E}-31$ & $6.4 \mathrm{E}-30$ & $1.7 \mathrm{E}-31$ \\
\hline-0.00495 & 0.6519 & $2 . \mathrm{E} 5$ & $-8.0 \mathrm{E} 6$ & $7.15 \mathrm{E} 6$ & $9.6 \mathrm{E}-30$ & $1.8 \mathrm{E}-30$ & $9.5 \mathrm{E}-30$ & $5.6 \mathrm{E}-31$ & $6.7 \mathrm{E}-30$ & $2.0 \mathrm{E}-31$ \\
\hline-0.01523 & 0.7103 & $4 . \mathrm{E} 5$ & $-1.1 \mathrm{E} 7$ & $8.24 \mathrm{E} 6$ & $1.2 \mathrm{E}-29$ & $1.1 \mathrm{E}-30$ & $1.2 \mathrm{E}-29$ & $4.8 \mathrm{E}-31$ & $1.2 \mathrm{E}-29$ & $2.3 \mathrm{E}-31$ \\
\hline-0.02002 & 0.6711 & $6 . \mathrm{E} 5$ & $-1.1 \mathrm{E} 7$ & $7.51 \mathrm{E} 6$ & $1.6 \mathrm{E}-29$ & $1.7 \mathrm{E}-30$ & $1.4 \mathrm{E}-29$ & $5.3 \mathrm{E}-31$ & $1.6 \mathrm{E}-29$ & $2.9 \mathrm{E}-31$ \\
\hline-0.02666 & 0.6643 & $8 . \mathrm{E} 5$ & $-1.2 \mathrm{E} 7$ & $7.38 \mathrm{E} 6$ & $1.8 \mathrm{E}-29$ & $1.7 \mathrm{E}-30$ & $1.8 \mathrm{E}-29$ & $5.4 \mathrm{E}-31$ & $1.8 \mathrm{E}-29$ & $3.4 \mathrm{E}-31$ \\
\hline-0.03327 & 0.6583 & $1 . \mathrm{E} 6$ & $-1.3 \mathrm{E} 7$ & $7.27 \mathrm{E} 6$ & $2.1 \mathrm{E}-29$ & $1.7 \mathrm{E}-30$ & $2.1 \mathrm{E}-29$ & $5.5 \mathrm{E}-31$ & $2.1 \mathrm{E}-29$ & $3.8 \mathrm{E}-31$ \\
\hline 0.36587 & 1.0968 & $1 . \mathrm{E} 7$ & 0.0 & $8.33 \mathrm{E} 7$ & $4.4 \mathrm{E}-29$ & $3.6 \mathrm{E}-31$ & $4.4 \mathrm{E}-29$ & $1.9 \mathrm{E}-31$ & $4.4 \mathrm{E}-29$ & $2.4 \mathrm{E}-31$ \\
\hline 3.65868 & 10.9684 & $1 . \mathrm{E} 8$ & 0.0 & $1.09 \mathrm{E} 9$ & $2.3 \mathrm{E}-29$ & $3.9 \mathrm{E}-32$ & $3.4 \mathrm{E}-29$ & $5.8 \mathrm{E}-32$ & $3.4 \mathrm{E}-29$ & $5.8 \mathrm{E}-32$ \\
\hline
\end{tabular}

\section{Axion phase effect}

Since the initial phase of the axion field is unknown, the phase $\varphi_{x}$ that appears in eq.(1.3) cannot be controlled in the experiment. However, the rate of the EDM precession angle strongly depends on the relative phase between the initial spin and axion phase $\varphi_{x}$. Fig. 2 shows the effect of initial phase on the vertical spin precession (EDM effect). The parameters used in the simulation are for the axion frequency of $10^{5} \mathrm{~Hz}$, which is shown in Table 1 . The spin tracking was done by 
integrating the following two equations for spin and velocity, respectively.

$$
\begin{gathered}
\frac{d \vec{s}}{d t}=\frac{e}{m} \vec{s} \times\left[\left(\frac{g}{2}-\frac{\gamma-1}{\gamma}\right) \vec{B}-\left(\frac{g}{2}-1\right) \frac{\gamma}{\gamma+1}(\vec{\beta} \cdot \vec{B}) \vec{\beta}-\left(\frac{g}{2}-\frac{\gamma}{\gamma+1}\right) \frac{\vec{\beta} \times \vec{E}}{c}+\frac{\eta}{2}\left(\vec{\beta} \times \vec{B}+\frac{\vec{E}}{c}-\frac{\gamma}{\gamma+1} \frac{\vec{\beta} \cdot \vec{E}}{c} \vec{\beta}\right)\right], \\
\frac{d \vec{\beta}}{d t}=\frac{e}{\gamma m}\left[\vec{\beta} \times \vec{B}+\frac{\vec{E}}{c}-\frac{\vec{\beta} \cdot \vec{E}}{c} \vec{\beta}\right] .
\end{gathered}
$$
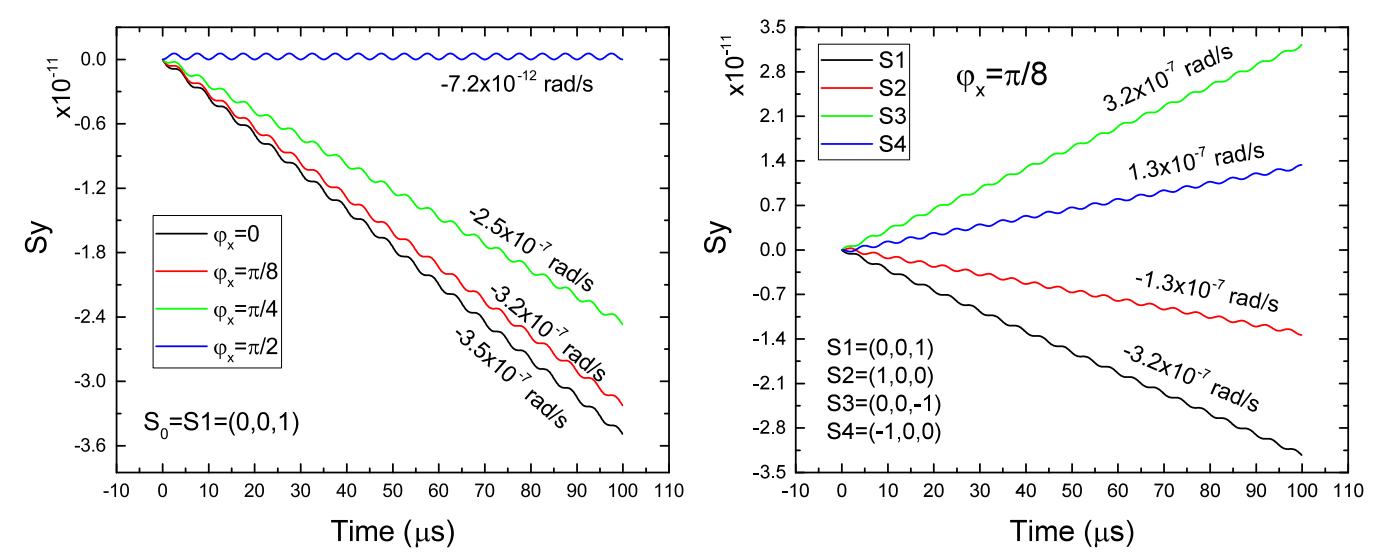

(a) Axion phase dependence of EDM precession (b) Spin polarization dependence of EDM prerates.

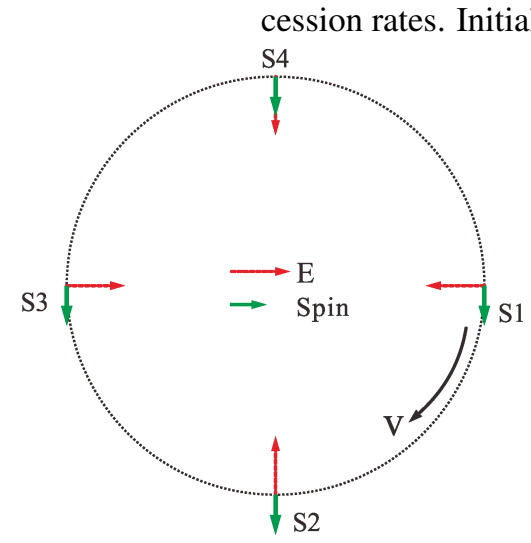

(c) Example of four orthogonal spin polarization settings.

Figure 2: Axion phases and EDM precession rates.

As can be seen in Fig. 2a, depending on the initial axion phases, the accumulated vertical EDM precession rates are different and random. However, this random axion phase issue can be resolved using two (or four) orthogonally set spin polarizations. An example of four orthogonally set polarizations are presented in Fig. 2c. Fig. 2b shows the effect of four orthogonally set spin polarizations on the $\omega_{E D M}$. From the measurement of individual spin polarization, one can calculate the total EDM precession rate using the relationship, $\omega_{E D M}=\sqrt{\omega_{E D M, S 1}^{2}+\omega_{E D M, S 2}^{2}}$, where $\omega_{E D M, S 1}$ and $\omega_{E D M, S 2}$ are two orthogonally set polarizations. The corresponding axion phase can be obtained by $\varphi_{x}=\arctan \left(\frac{\omega_{E D M, S 2}}{\omega_{E D M, S 1}}\right)$. As can be seen in the example shown in Fig. 2b, the S1 
and S3 states have large precession rates $\left(3.2 \times 10^{-7} \mathrm{rad} / \mathrm{s}\right)$ for the axion phase of $\varphi_{x}=\pi / 8$, and the precession directions are opposite to each other. On the other hand, note that the other two polarizations S2 and S4 have smaller $\omega_{E D M}$ than the two counter part of polarizations (S1, S3). In any case, the actual precession rate $\omega_{E D M}$ can be calculated using the formula shown above.

\section{Summary and conclusion}

As a candidate for dark matter, the axion has been the target of extensive searches using microwave cavities and other methods. The fact that the axion-gluon coupling can produce an oscillating EDM in nucleons led to the novel idea of measuring the oscillating EDM in hadronic particles like the proton and deuteron. We propose using the storage ring technique to measure the axion induced oscillating EDM at the resonance conditions between the axion frequency and $g-2$ spin precession frequency.

In this study, we calculated the electric field and magnetic field that are required for the resonance conditions. With the experimental conditions, we estimated the achievable sensitivities, and the result shows the experiment is more sensitive than the planned static EDM measurement $\left(10^{-29} e \cdot \mathrm{cm}\right)$ by at least by one order of magnitude, $\leq 10^{-30} e \cdot \mathrm{cm}$. This sensitivity is achieved if we assume that we know the axion frequency and spend all the experimental time at one frequency value. At very low frequencies, $f_{a x}<1 \mathrm{mHz}$, one can search for the axion with the sensitivities of $10^{-31}-10^{-32} e \cdot \mathrm{cm}$ using the frozen spin method, without knowing the axion frequencies.

A wide range of frequencies $\left(10^{-9} \mathrm{~Hz}-100 \mathrm{MHz}\right)$ of axion dark matter can be searched by using both deuterons and protons in the same storage ring. Even though the proposed method does not reach the estimated sensitivity needed to reach the theoretical models of axion dark matter induced oscillating EDM, it promises to be one of the most sensitive ways to look for axions over a wide frequency range.

\section{Acknowledgement}

This work was supported by IBS-R017-D1-2018-a00 of the Republic of Korea.

\section{References}

[1] P. Sikivie, Experimental tests of the "invisible" axion, Phys. Rev. Lett. 51, 1415-1417 (1983).

[2] Peter W. Graham and Surjeet Rajendran, Axion dark matter detection with cold molecules, Phys. Rev. D 84, 055013 (2011).

[3] Peter W. Graham and Surjeet Rajendran, New observables for direct detection of axion dark matter, Phys. Rev. D 88,035023 (2013).

[4] Peter W. Graham and Surjeet Rajendran. New Ob- servables for Direct Detection of Axion Dark Matter. Phys. Rev., D88:035023, 2013.

[5] V. Anastassopoulos et al., A Proposal to Measure the Proton Electric Dipole Moment with $10^{-} 29 e \cdot \mathrm{cm}$ Sensitivity by the Storage Ring EDM Collaboration., Available at https://www.bnl.gov/edm/files/pdf/Proton_EDM_proposal_20111027_final.pdf. 
[6] V. Anastassopoulos et al., A storage ring experiment to detect a proton electric dipole moment. Review of Scientific Instruments, 87, 115116 (2016).

[7] V. Bargmann, Louis Michel, and V. L. Telegdi, Precession of the polarization of particles moving in a homogeneous electromagnetic

field, Phys. Rev. Lett., 2, 435 (1959).

[8] John David Jackson, Classical electrodynamics. Wiley, New York, NY, 3rd edition, 1999.

[9] Gerald G. Ohlsen and P.W. Keaton, Techniques for measurement of spin-1/2 and spin-1 polarization analyzing tensors, Nuclear Instruments and Methods 109, 41 (1973). 